\title{
Whole-genome analysis of Alu repeat elements reveals complex evolutionary history
}

\author{
Alkes L. Price, ${ }^{1}$ Eleazar Eskin, and Pavel A. Pevzner \\ Department of Computer Science and Engineering, University of California-San Diego, La Jolla, California 92093-0114, USA
}

\begin{abstract}
Alu repeats are the most abundant family of repeats in the human genome, with over 1 million copies comprising $10 \%$ of the genome. They have been implicated in human genetic disease and in the enrichment of gene-rich segmental duplications in the human genome, and they form a rich fossil record of primate and human history. Alu repeat elements are believed to have arisen from the replication of a small number of source elements, whose evolution over time gives rise to the 31 Alu subfamilies currently reported in Repbase Update. We apply a novel method to identify and statistically validate 213 Alu subfamilies. We build an evolutionary tree of these subfamilies and conclude that the history of Alu evolution is more complex than previous studies had indicated.
\end{abstract}

[Supplemental material is available online at www.genome.org.]

Alu repeats are a family of short interspersed elements (SINEs) that replicate via LINE-mediated reverse transcription of an RNA polymerase III transcript (Rogers 1983; Mathias et al. 1991; Dewannieux et al. 2003). Each Alu element is roughly $280 \mathrm{bp}$ long, followed by a poly-A tail of variable length. Thus, the more than 1 million Alu elements comprise roughly $10 \%$ of the human genome (International Human Genome Consortium 2001). Although Alu repeats have no known biological function (Schmid 2003), the study of the Alu repeat family has many ramifications. Alu insertions and $A l u$-mediated unequal recombination contribute to a significant proportion of human genetic disease (Deininger and Batzer 1999). Alu-mediated unequal recombination is believed to be responsible for the enrichment of gene-rich segmental duplications in humans versus other sequenced organisms (Bailey et al. 2003). Alu repeats have been used to study the history of substitution patterns in the human genome (Arndt et al. 2003), and polymorphic Alu insertions have been used as markers to determine genetic distances between human subpopulations (Watkins et al. 2003). Recently, a phylogenetic analysis of Alu elements belonging to the Alu Ye5 subfamily has provided the strongest evidence yet that the chimp is humans' closest living relative (Salem et al. 2003). Virtually all areas of Alu research rely on the classification of Alu subfamilies, and this paper provides strong evidence that the existing classification is incomplete.

Early analyses, prior to the assembly of the human genome, identified a small number of Alu subfamilies, each characterized by a few diagnostic positions in its consensus sequence (Willard et al. 1987; Britten et al. 1988; Deininger and Slagel 1988; Jurka and Smith 1988; Quentin 1988; Matera et al. 1990a; Batzer and Deininger 1991; Jurka and Milosavljevic 1991). These analyses led to the conjecture that all Alu repeat elements have arisen from the replication of either "a single master gene" (Shen et al. 1991), or "an extremely small group of master genes" (Deininger et al. 1992). The conjecture of a single master gene was shown to be incorrect (Matera et al. 1990b; Jurka and Milosavljevic 1991;

\section{'Corresponding author.}

E-mail aprice@cs.ucsd.edu; fax (858) 534-7029.

Article and publication are at http://www.genome.org/cgi/doi/10.1101/ gr.2693004.
Leeflang et al. 1992), but it remains widely believed that "only a few human Alu elements ... seem to be retrotransposition competent" (Batzer and Deininger 2002). In this paper, we will refer to Alu elements that have (or previously had) the ability to replicate as source elements (Matera et al. 1990b); we note that many different definitions of the terms "source gene" and "master gene" have been used previously (Matera et al. 1990b; Shen et al. 1991; Deininger et al. 1992; Deininger and Batzer 1995). As a source element evolves over time, it may produce a lineage of more than one subfamily (Leeflang et al. 1993). The Repbase Update database (Jurka 1998, 2000) keeps a record of known Alu subfamilies, each defined by a consensus sequence. New Alu subfamilies have been added to the database as recently as 2002, and there are 31 Alu subfamilies currently reported in the database. ${ }^{2}$

With the sequence of the human genome now assembled (International Human Genome Consortium 2001), Alu repeats can be analyzed on a genome-wide scale. In our genome-wide analysis, we have identified and statistically validated $213 \mathrm{Alu}$ subfamilies, each defined by a consensus sequence. Our novel method recursively splits subfamilies whose members fail a statistical uniformity test. After identifying Alu subfamilies, we built an evolutionary tree of these subfamilies. Our evolutionary tree describes the path of evolution from AluJ subfamilies to AluS subfamilies, and from AluS subfamilies to AluY subfamilies, at a much finer granularity than previous analyses. Our evolutionary tree also contains a large number of completely new branches. We conclude that the history of Alu evolution is more complex than previous studies had indicated.

The existing set of repeat subfamily identification algorithms is quite limited. Recent algorithms such as RepeatMasker (A.F.A. Smit and P. Green, http://repeatmasker.org), REPuter (Kurtz et al. 2000), RepeatFinder (Volfovsky et al. 2001), RECON (Bao and Eddy 2002), and RepeatGluer (Pevzner et al. 2004) are successful at finding individual repeat elements or identifying repeat families, but they do not address the problem of identifying very similar subfamilies of a repeat family. The only previous algorithm for identifying repeat subfamilies that we are aware of

${ }^{2}$ The Alu section of Repbase Update also contains 3 additional subfamilies, each roughly $140 \mathrm{bp}$ long, representing monomeric ancestors that pre-date modern dimeric Alu repeats and are thus outside the scope of this study. 
Table 1. Finding the Ya5 subfamily in the set of all Alu elements (A) All Alus: nucleotide frequencies

\begin{tabular}{lccccc}
\hline & $\mathbf{1}$ & $\mathbf{2}$ & $\mathbf{3}$ & $\mathbf{4}$ & $\mathbf{5}$ \\
\hline $\mathrm{A}$ & 0.01 & $\underline{0.02}$ & $\underline{0.06}$ & 0.03 & 0.31 \\
$\mathrm{C}$ & $\underline{0.02}$ & $\mathbf{0 . 8 7}$ & 0.01 & $\mathbf{0 . 6 3}$ & $\underline{0.02}$ \\
$\mathrm{G}$ & 0.00 & 0.02 & $\mathbf{0 . 9 1}$ & 0.03 & $\mathbf{0 . 6 4}$ \\
$\mathrm{T}$ & $\mathbf{0 . 9 6}$ & 0.08 & 0.01 & $\underline{0.31}$ & 0.02 \\
\hline
\end{tabular}

(B) $k$-means clustering

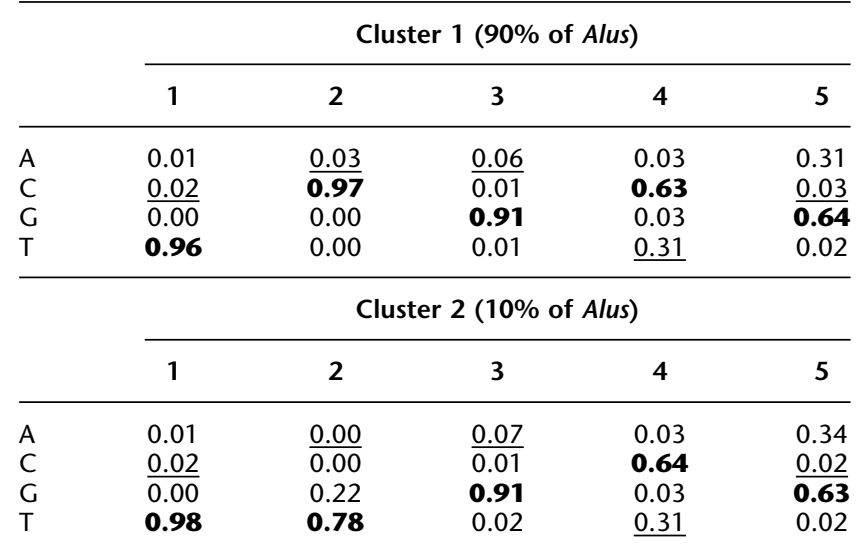

(C) All Alus: binucleotide frequencies relative to expected

\begin{tabular}{lcccccccccc}
\hline & $\mathbf{1 , 2}$ & $\mathbf{1 , 3}$ & $\mathbf{1 , 4}$ & $\mathbf{1 , 5}$ & $\mathbf{2 , 3}$ & $\mathbf{2 , 4}$ & $\mathbf{2 , 5}$ & $\mathbf{3 , 4}$ & $\mathbf{3 , 5}$ & $\mathbf{4 , 5}$ \\
\hline A,A & 1 & 1 & 1 & 1 & $\frac{5}{1}$ & 1 & 1 & 1 & 1 & 1 \\
A,C & 1 & 1 & 1 & 1 & 1 & $\frac{12}{1}$ & 1 & $\frac{5}{1}$ & 1 \\
A,G & 1 & 1 & 1 & 1 & 1 & 1 & 1 & 1 & 1 & 1 \\
A,T & 1 & 1 & 1 & 1 & 1 & $\frac{2}{1}$ & 1 & $\frac{1}{1}$ & 1 & 1 \\
C,A & 12 & $\frac{5}{1}$ & 1 & 1 & 1 & 1 & 1 & 1 & 1 & 1 \\
C,C & 1 & 1 & 1 & $\frac{11}{1}$ & 1 & 1 & 1 & 1 & 1 & 1 \\
C,G & 1 & 1 & 1 & 1 & 1 & 1 & 1 & 1 & 1 & 1 \\
C,T & 1 & 1 & 2 & 1 & 1 & 1 & 1 & 1 & 1 & 1 \\
G,A & 1 & 1 & 1 & 2 & 2 & 1 & 1 & 1 & 1 & 1 \\
G,C & 1 & 1 & 1 & 1 & 1 & 1 & 1 & 1 & 1 & 1 \\
G,G & 1 & 1 & 1 & 1 & 1 & 1 & 1 & 1 & 1 & 1 \\
G,T & 1 & 1 & 1 & 1 & 1 & 1 & 1 & 1 & 1 & 1 \\
T,A & 1 & 1 & 1 & 1 & 1 & 1 & 1 & 1 & 1 & 1 \\
T,C & 1 & 1 & 1 & 1 & 1 & 1 & 1 & 1 & 1 & 2 \\
T,G & 1 & 1 & 1 & 1 & 1 & 1 & 1 & 1 & 1 & 1 \\
T,T & 1 & 1 & 1 & 1 & 1 & 1 & 1 & 1 & 1 & 1
\end{tabular}

\section{(D) Our algorithm}

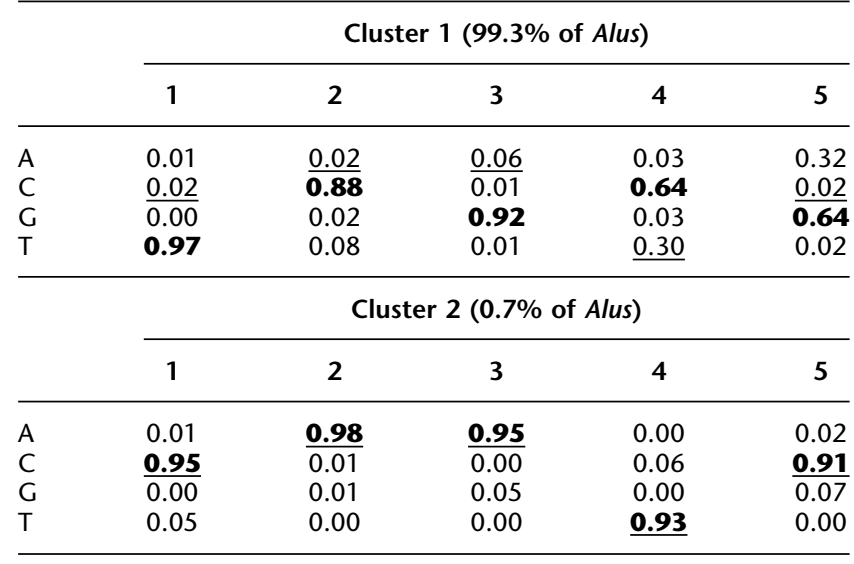

taining all of these $A l u$ elements, we recursively split subfamilies whose members fail a statistical uniformity test. We first split subfamilies containing overrepresented pairs of nonconsensus nucleotide values to build a scaffold of the space of Alu subfamilies, performing an additional validation step to verify that the union of two or more subfamilies fails the uniformity test, using a $P$-value threshold of 0.001 (see Methods). The resulting scaffold contains 60 Alu subfamilies. We then use the resulting calibration of $A l u$ mutation rates at each position to split subfamilies containing overrepresented individual mutations, again using a $P$-value threshold of 0.001 (see Methods). This procedure identifies 153 additional subfamilies. Thus, we identify a total of 213 Alu subfamilies. The size of each subfamily ranges from roughly 50 to $60,000 \mathrm{Alu}$ elements, with most subfamilies containing at least a few hundred elements. $P$-values for each subfamily range from below $1 \mathrm{e}^{-6000}$ to near our $P$-value threshold of 0.001 , with most subfamilies having a $P$-value below $1 \mathrm{e}^{-40}$.

We have chosen a sample of 12 Alu subfamilies identified by our algorithm to describe here, including six subfamilies that are currently reported in Repbase Update (Jurka 1998; Jurka 2000) and six novel subfamilies from our scaffold of $60 \mathrm{Alu}$ subfamilies. We list the aligned consensus sequences of these 12 subfamilies in Figure 2, and their sizes and $P$-values in Table 2 . Consensus sequences for all 213 subfamilies identified by our algorithm are listed elsewhere (see online Supplemental materials).

We build an evolutionary tree of Alu subfamilies that describes the history of $A l u$ evolution. In contrast to the typical scenario in phylogenetic tree reconstruction in which the input data contains only external nodes of the tree, our Alu subfamilies may be either internal or external nodes of the evolutionary tree; this is because Alu repeat elements in the genome give us a fossil record of $A l u$ subfamilies from the past as well as the present. Thus, traditional methods for phylogenetic tree reconstruction are not applicable here. We instead define the evolutionary tree of Alu subfamilies to be their Minimum Spanning Tree (Kruskal 1956) (see Methods). The resulting evolutionary tree of the 31 subfamilies currently reported in Repbase Update is displayed in Figure 3 , and the evolutionary tree of the 213 subfamilies we have identified is displayed in Figure 4.

As an Alu source element evolves over time, it may produce a lineage of more than one Alu subfamily (thus the number of Alu subfamilies may exceed the number of Alu source elements) but these subfamilies must correspond to a single path in the Alu evolutionary tree. Thus, by counting the number of leaves in the tree, we obtain a lower bound on the number of source elements. Our evolutionary tree of 213 subfamilies implies that there are at least $143 \mathrm{Alu}$ source elements. In contrast, the evolutionary tree of the 31 subfamilies currently reported in Repbase Update implies the existence of only 14 Alu source elements.

For simplicity, we considered only the 5 Alu positions with diagnostic mutations in the Ya5 subfamily (positions 91, 98, 146, 175, and 238, assuming that positions of the AluSx consensus sequence are labeled from 1 to 282). In each table, entries corresponding to the Ya5 consensus are underlined. In (A), entries corresponding to the Alu consensus are indicated in boldface type. In (B) and (D), entries corresponding to the consensus of each respective cluster are indicated in bolface type. (A) The nucleotide frequency profile of all Alus. (B) Frequency profiles for the 2 clusters returned by $k$-means clustering with $k=2$, which does not find the Ya5 subfamily. (C) Ratio of actual versus expected biprofile frequencies at each pair of positions, rounded to the nearest integer. (D) Frequency profiles for the 2 clusters found by our algorithm, which finds the Ya5 subfamily. 
Price et al.

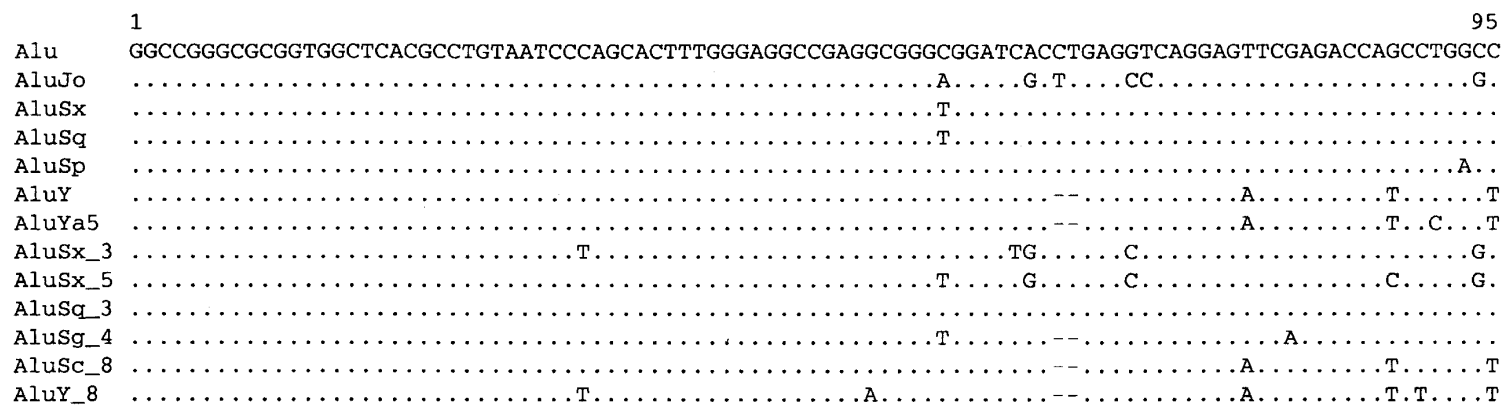

96

189

Alu AACATGGTGAAACCCCGTCTCTACTAAAAATACAAAAA-TTAGCCGGGCGTGGTGGCGCGCGCCTGTAATCCCAGCTACTCGGGAGGCTGAGGCA

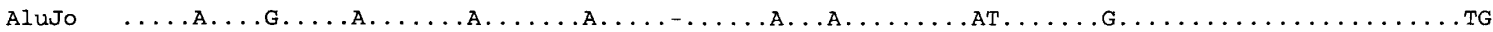

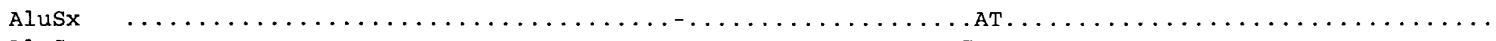

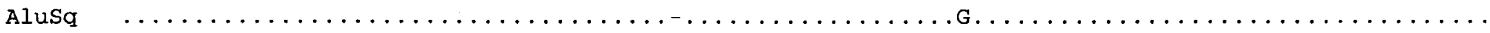

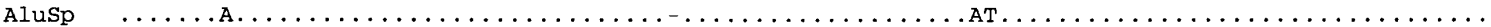

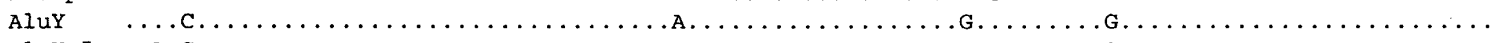

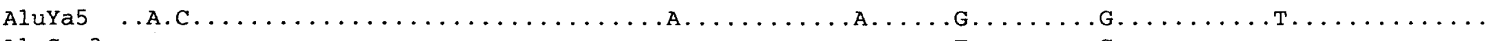

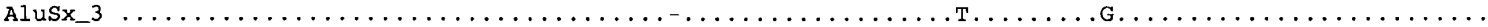

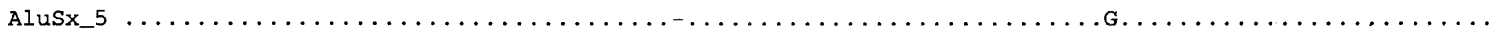

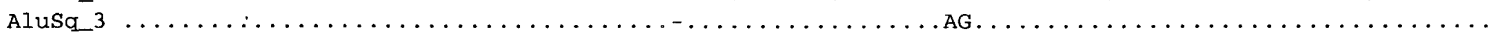

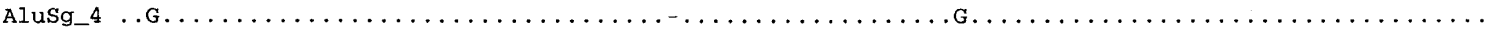

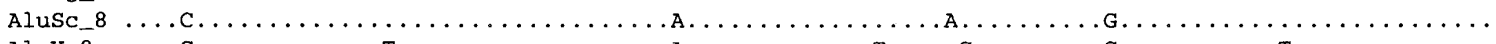

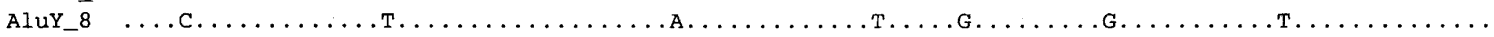

$190 \quad 282$

Alu GGAGAATCGCTTGAACCCGGGAGGCGAGGTTGCAGTGAGCCGAGATCGCGCCACTGCACTCCAGCCTGGGCGACA-GAGCGAGACTCCGTCTC

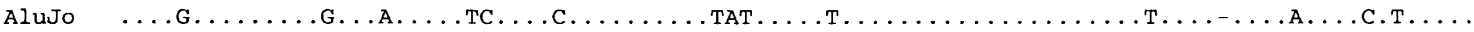

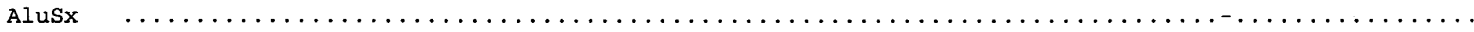

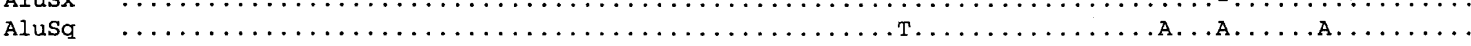

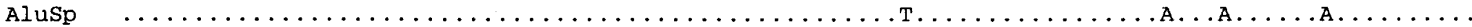

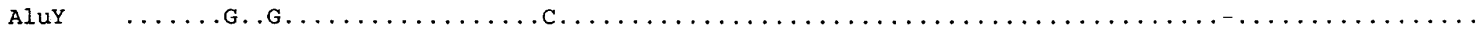

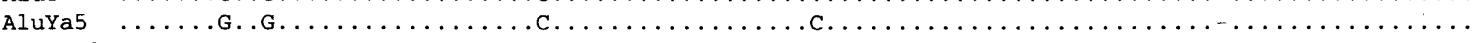

Alusx_3 .....

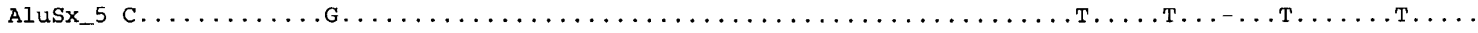

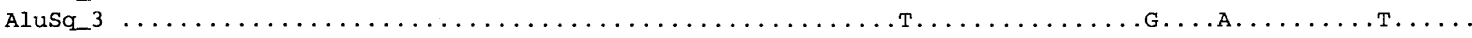

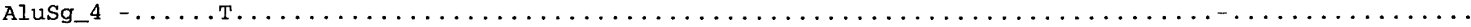

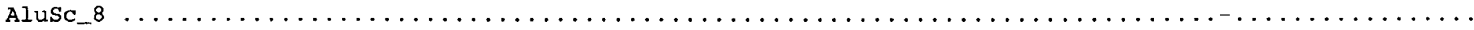

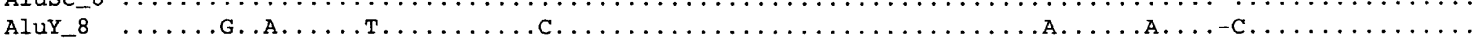

Figure 2. Aligned consensus sequences of selected subfamilies. (Top) The consensus sequence of the entire Alu family, with positions labeled from 1 to 282. (Middle) The consensus sequences of six Alu subfamilies we identified that are currently reported in Repbase Update: Alulo, AluSx, AluSq, AluSp, AluY, and AluYa5; the few discrepancies between our consensus sequences and the consensus sequences reported in Repbase Update occur mostly at $\mathrm{CpG}$ dinucleotide positions, which are ill-determined because of frequent mutation. (Bottom) The consensus sequences of six Alu subfamilies we identified that are not currently reported in Repbase Update: AluSx_3, AluSx_5, AluSq_3, AluSg_4, AluSc_8, and AluY_8.

\section{Discussion}

We have identified a total of 213 Alu subfamilies. Our evolutionary tree of these subfamilies describes the path of evolution from AluJ subfamilies to AluS subfamilies, and from AluS subfamilies to AluY subfamilies, at a much finer granularity than previous analyses. For example, the path of evolution from AluJ subfamilies to AluS subfamilies includes the novel AluSx_3 subfamily, ${ }^{4}$ and the path of evolution from AluS subfamilies to AluY subfamilies includes the novel AluSc_8 subfamily. Our evolutionary tree also contains a large number of completely new branches, revealing a complex evolutionary history. The abundance of previously undiscovered subfamilies in the earlier phases of Alu evolution is particularly striking; because their elements have mutated significantly from the consensus sequence, most of these subfamilies can only be detected by a rigorous whole-genome analysis. Looking in detail at the remaining novel subfamilies listed in Figure 2, the novel AluSx_5 subfamily, a descendant of the

${ }^{4}$ To adhere to the existing nomenclature (Batzer et al. 1996), we name our subfamilies by assigning them to existing Repbase Update subfamilies, e.g., AluSx, AluSx_2, AluSx_3, etc.
AluSx_3 subfamily, can be viewed as a cousin of the AluSx subfamily; the novel AluSq_3 and AluSg_4 subfamilies are moderately large and quite different from their ancestors (AluSq and AluSg) in Repbase Update; and the novel AluY_8 subfamily is very different from its ancestor (AluY) in Repbase Update.

Our results are partially, but not entirely, consistent with existing theories of Alu evolution. Our lower bound of $143 \mathrm{Alu}$ source elements is much larger than previous studies had indicated; we further speculate that there are many Alu subfamilies that we have not identified, either because they are not statistically discernible or because of limitations in our algorithm. We conjecture that there may be thousands of Alu subfamilies, and thousands of Alu source elements. Previous studies had suggested a mostly linear Alu evolution pattern in which most parallel subfamily formations involve very low copy number or short-lived subfamilies (Deininger and Batzer 1995); Figure 4 does contain many short, low-copy-number branches but also contains many major branches that have propagated a large number of Alu copies. Our results are consistent with a model in which a large number of Alu copies are themselves source elements, replicating at widely varying rates; however, these hundreds (or perhaps

\section{Genome Research}

www.genome.org 
thousands) of Alu source elements represent a tiny fraction of the more than 1 million Alu elements. It remains clear that the majority of $A l u$ elements are not retrotransposition competent; a common explanation for this is that appropriate upstream sequence is required for efficient Alu transcription (Ullu and Weiner 1985). The abundance of short branches in the Alu evolutionary tree suggests that many source elements are retrotransposition competent for only a short time, perhaps because mutations to the CpG dinucleotides of an Alu source element, or to its poly-A tail, may eliminate their retrotransposition capability (Batzer and Deininger 2002).

Our algorithm has several known limitations. For technical reasons, we exclude insertion/deletion mutations, frequent $\mathrm{CpG}$ mutations, and mutations to nucleotide values already present in other subfamilies as a means of identifying new subfamilies (see Methods), making subfamilies characterized by these mutations difficult to identify. In addition, the partition of the set of Alu elements into statistically distinguishable subfamilies need not be unique, and there may exist subfamilies whose elements are distributed across more than one member of our partition, making them difficult to identify. There is no immediate fix to these limitations in our algorithm; they are important directions of our ongoing research. Because of these limitations, our algorithm identifies only 19 of the 31 subfamilies currently reported in Repbase Update. Combining the 213 Alu subfamilies identified by our algorithm with the 12 Alu subfamilies in Repbase Update not identified by our algorithm (which each belong to minor branches of the AluY subfamily), there are a total of 225 previously and presently identified Alu subfamilies. A complete list of these subfamilies is given in the Supplemental materials.

An improved characterization of Alu subfamilies and their evolutionary history will benefit numerous applications, such as analysis of segmental duplications induced by Alu recombination (Bailey et al. 2003), and phylogenetic inference using Alus. Recently, a phylogenetic analysis of Alu elements in the Ye5 subfamily has provided the strongest evidence yet that the chimp is humans' closest living relative (Salem et al. 2003). We hope that the novel Alu subfamilies we have identified may lead to phylogenetic inferences involving other primate species. Furthermore, our methods can be used to identify subfamilies of other repeat families in non-primate species, an open problem. SINE elements

Table 2. Sizes and $P$-values of selected subfamilies

\begin{tabular}{lrl}
\hline Subfamily & Size & $P$-value \\
\hline Alujo & 7,266 & $8 \mathrm{e}^{-1841}$ \\
AluSx & 39,724 & $6 \mathrm{e}^{-4770}$ \\
AluSq & 4,035 & $2 \mathrm{e}^{-62}$ \\
AluSp & 28,063 & $7 \mathrm{e}^{-4520}$ \\
AluY & 27,023 & $2 \mathrm{e}^{-6924}$ \\
AluYa5 & 3,257 & $4 \mathrm{e}^{-2813}$ \\
AluSx_3 & 3,292 & $8 \mathrm{e}^{-1841}$ \\
AluSx_5 & 401 & $3 \mathrm{e}^{-150}$ \\
AluSq_3 & 1,956 & $2 \mathrm{e}^{-779}$ \\
AluSg_4 & 1,904 & $1 \mathrm{e}^{-679}$ \\
AluSc_8 & 9,588 & $1 \mathrm{e}^{-5959}$ \\
AluY_8 & 107 & $1 \mathrm{e}^{-48}$ \\
\hline
\end{tabular}

We list the size and $P$-value for each of the 12 subfamilies whose aligned consensus sequences are listed in Figure 2. Some Repbase Update subfamilies, particularly the AluSq subfamily, contain fewer elements in our allocation of Alu elements to subfamilies than in the allocation of Alu elements to Repbase Update subfamilies only, because many elements have been reallocated to neighboring subfamilies not in Repbase Update.

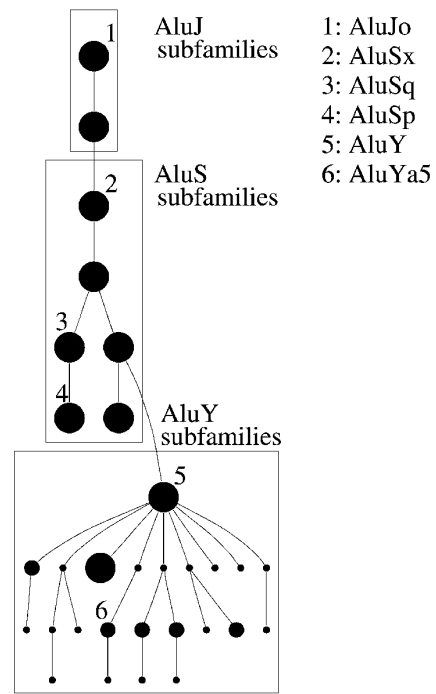

Figure 3. Evolutionary tree of the 31 subfamilies currently reported in Repbase Update. (Large nodes) Subfamilies with more than 10,000 elements; (medium nodes) 1000 to 10,000 elements; (small nodes) less than 1000 elements. Each of the 6 Repbase Update subfamilies listed in Figure 2 is labeled. The Alu], AluS, and AluY classes of subfamilies are contained in boxes.

have already been used to make phylogenetic deductions about cetartiodactyls (Nikaido et al. 1999) and cichlid fish (Takahashi et al. 2001), and an improved characterization of repeat subfamilies may aid such efforts in the future.

\section{Methods}

We generated a data set of $A l u$ elements via a BLAST search (Tatusova and Madden 1999) of Build 34 of the human genome (International Human Genome Consortium 2001) against the AluSx consensus sequence reported in Repbase Update (Jurka 1998, 2000); equivalently, this data set can be generated using RepeatMasker (A.F.A. Smit and P. Green, http://repeatmasker. org). We multiply aligned the Alu elements in our data set by tabulating the nucleotide value of each Alu element at each position of the AluSx consensus sequence, with insertions recorded separately. Because our method assumes that the nucleotide value of each Alu element at each position is known, we excluded Alu elements whose alignment to AluSx is missing more than 5 bases at the beginning or end. After imposing this restriction, there were roughly 480,000 full-length Alu elements in our data set.

We split subfamilies containing overrepresented pairs of non-consensus nucleotide values as follows. Let $\mu_{1}$ and $\mu_{2}$ be two mutations from the consensus sequence. Let $N$ be the number of repeat elements in the subfamily, $N_{\mathrm{i}}$ be the number of repeat elements with mutation $i$ (for $i=1,2$ ), and $N_{12}$ be the number of repeat elements with both mutations. If the two mutations are unlinked, we expect

$$
N_{12} \approx \frac{N_{1} N_{2}}{N}
$$

If

$$
N_{12}>\frac{N_{1} N_{2}}{N}
$$




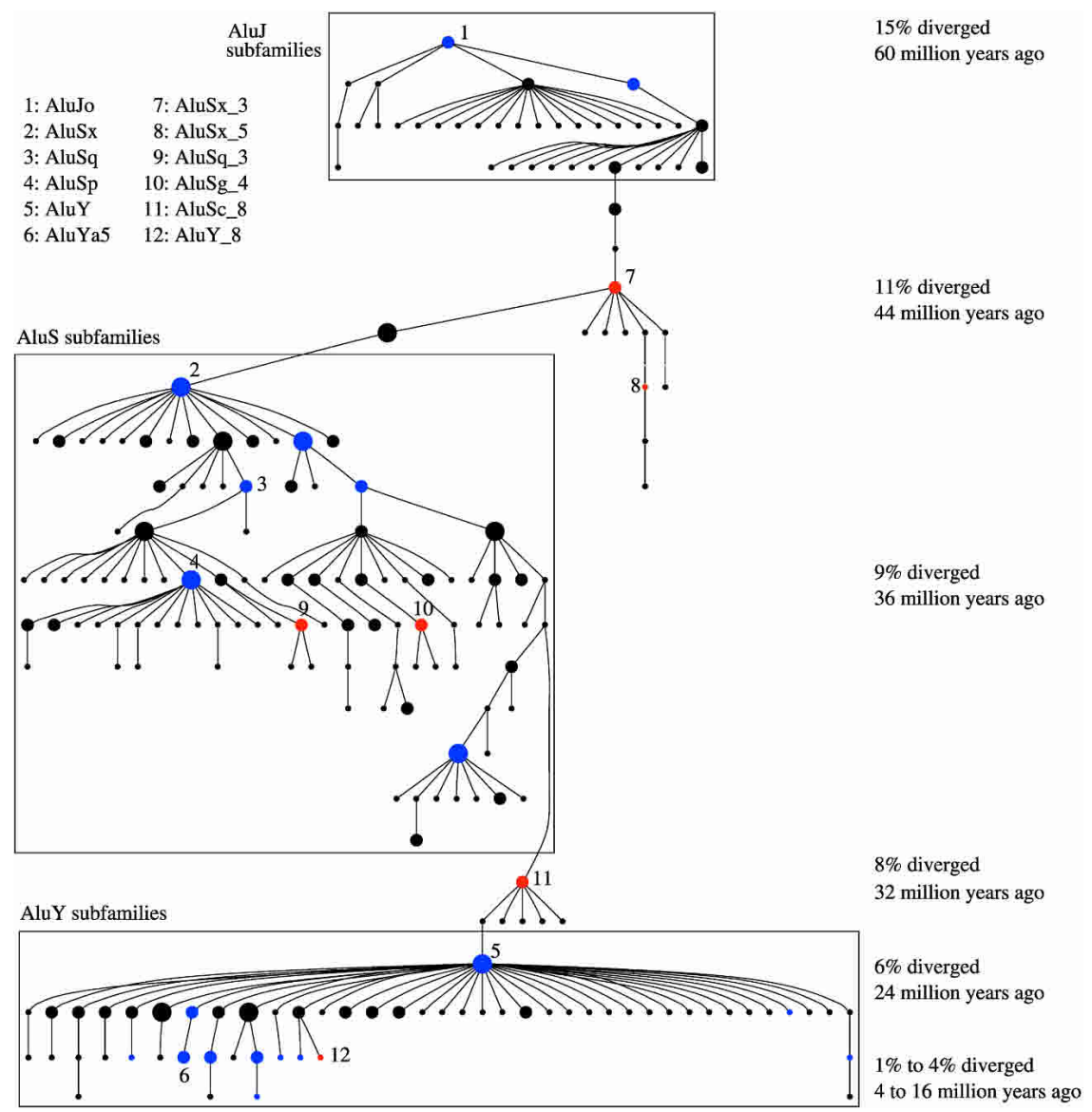

Figure 4. Evolutionary tree of the 213 subfamilies we identified. (Large nodes) Subfamilies with more than 10,000 elements; (medium nodes) 1000 to 10,000 elements; (small nodes) less than 1000 elements. Subfamilies listed in Repbase Update are colored blue, and the 6 novel subfamilies listed in Figure 2 are colored red. Each of the subfamilies listed in Figure 2 is labeled. A rendition of this tree with every node labeled is available in the Supplementary materials online. The Alu], AluS, and AluY classes of subfamilies are contained in boxes; not all subfamilies fit into one of these classes. A timeline roughly depicting the average divergence of each subfamily from its consensus sequence and the approximate age obtained by applying a constant scaling factor of 4 million years per $1 \%$ divergence from consensus sequence are included at right.

then the ratio of the actual versus expected frequency of both mutations, which equals

$$
\frac{N_{12} N}{N_{1} N_{2}}
$$

quantifies the extent of the linkage (as in Table 1C). We computed a $P$-value for the linkage using a nonparametric $P$-value computation, which makes no assumptions about the underlying probability distributions: the probability of at least $N_{12}$ repeat elements with both mutations occurring by chance is

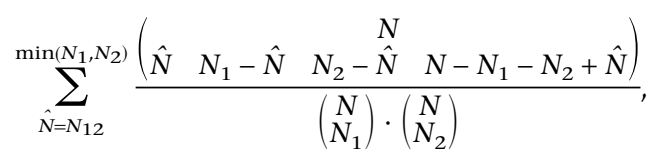

where the denominator represents the total number of ways to allocate the two mutations to the sequences, and the multinomial coefficient in the numerator represents the number of allocations with exactly $\hat{N}$ sequences containing both mutations. The above expression, which we denote $\xi_{\mu_{1}, \mu_{2}}$ is a $P$-value for the observed linkage of $\mu_{1}$ and $\mu_{2}$ under the null hypothesis of uniformity. Thus, $\xi=\min _{\mu_{1}, \mu_{2}} \xi_{\mu_{1}, \mu_{2}}$ gives an overall $P$-value for the uniformity of the subfamily. If $\xi$ is below a threshold, which we set at 0.001 , the subfamily fails the uniformity test and we split it accordingly. We ensure that the assignment of all Alu repeat elements to one of the resulting subfamilies is consistent, that is, that the consensus sequence defining each subfamily matches the consensus sequence of its members: At each step of the algorithm, we iteratively reassign all $A l u$ repeat elements to subfamilies and recompute the consensus of each subfamily, until this process converges.

To insure the validity of our $P$-value computation, we addressed several important details. First, for a given subfamily, we computed $\xi_{\mu_{1}, \mu_{2}}$ for many different pairs of mutations $\left(\mu_{1}, \mu_{2}\right)$. To compensate for the possibility of obtaining a low value of $\xi_{\mu_{1}, \mu_{2}}$ by chance, we applied a Bonferroni correction, multiplying each $\xi_{\mu_{1}, \mu_{2}}$ by the number of pairs $\left(\mu_{1}, \mu_{2}\right)$ tested. To verify that this Bonferroni correction was sufficient, we simulated a uniform data set from the probability profile of all Alu repeat elements and observed that $P$-values computed from this data set were all greater than 1, after the Bonferroni correction. Second, in our application to Alu repeat subfamilies, a single source element might produce copies over a long span of time, thus producing older copies with many mutations from the consensus and newer copies with fewer mutations from the consensus. This would bias any two mutations $\mu_{1}$ and $\mu_{2}$ into being linked, because $A l u$ copies with mutation $\mu_{1}$ would be likely to be older and thus have mutation $\mu_{2}$ also. Routine calculations showed that this effect could bias the number of repeat elements with both $\mu_{1}$ and $\mu_{2}$ upwards by a factor of up to $4 / 3$. We modified the computation of $\xi_{\mu_{1}, \mu_{2}}$ to account for this bias. Third, because insertion/deletion mutations violate our assumption that distinct positions mutate independently and cause further technical problems, we excluded the case of two indel mutations in our $P$-value computation and imposed a minimum distance of 10 nucleotides between any two mutations $\mu_{1}$ and $\mu_{2}$. Fourth, the Alu consensus sequence contains many CpG dinucleotides, which are highly prone to methylation and subsequent mutation to TpG or CpA (Labuda and Striker 1989). These mutations violate our independence assumption and complicate the computation of the correct consensus sequence (e.g., a dinucleotide with frequent occurrences of both TpG and CpA has correct consensus $\mathrm{CpG}$, but its consensus computed under the independence assumption may equal TpG or $\mathrm{CpA}$ ). Thus, we excluded $\mathrm{CpG} \rightarrow \mathrm{TpG}$ and $\mathrm{CpG} \rightarrow \mathrm{CpA}$ mutations and the reverse of these mutations in our $P$-value computation. Fifth, we excluded mutations to nucleotide values already present in other subfamilies; this very conservative restriction is necessary to avoid falsely assigning mosaic Alu elements formed by Alu-Alu

\section{Genome Research}

www.genome.org 
recombination to separate subfamilies. Sixth, we excluded pairs of mutations $\left(\mu_{1}, \mu_{2}\right)$ for which the number $N_{12}$ of repeat elements is $<50$, thus imposing a minimum subfamily size of 50 ; this insures that a small number of copies of a repeat element formed by segmental duplication will not be assigned to a separate subfamily.

After we finished splitting all subfamilies containing overrepresented pairs of nonconsensus nucleotide values, we verified that the union of two or more subfamilies fails the uniformity test, otherwise we merged subfamilies accordingly. Because it is not computationally feasible to perform a separate check for every possible union of two or more subfamilies, we checked only the unions formed when building the Minimum Spanning Tree (see below). We defined the $P$-value for each subfamily in the resulting scaffold as the $P$ - value obtained by testing the uniformity of the union of that subfamily with its parent in the Minimum Spanning Tree.

After building a scaffold of Alu subfamilies, we calibrated $A l u$ mutation rates at each position from a given consensus value to every other value by averaging over the elements of all subfamilies in the scaffold that have that consensus value; the mutation rates were computed relative to the overall divergence of a subfamily from its consensus sequence, which serves as our proxy for its age. This calibration allowed us to split subfamilies containing overrepresented individual mutations. We computed $P$-values using a simple binomial test, and split each subfamily containing an overrepresented individual mutation with a $P$-value below 0.001 , assigning this $P$-value to the newly created subfamily. As before, we applied an appropriate Bonferroni correction, excluded insertion/deletion and frequent CpG mutations, and imposed a minimum subfamily size of 50 . We excluded mutations to nucleotide values already present in other subfamilies, unless the nucleotide value was present in an adjacent subfamily of the Minimum Spanning Tree of Alu subfamilies (see below), in which case splitting with respect to that mutation would simply add an intermediary subfamily.

Because traditional methods for phylogenetic tree reconstruction are not applicable here, we defined the evolutionary tree of $A l u$ subfamilies to be their Minimum Spanning Tree, that is, the tree with Alu subfamilies as nodes that minimizes the sum of edge distances. We built the Minimum Spanning Tree of Alu subfamilies using Kruskal's algorithm (Kruskal 1956), iteratively connecting the two closest subfamilies in different connected components of the tree. We defined the distance between two subfamilies to be the Hamming distance between their consensus sequences, ignoring $\mathrm{CpG} \rightarrow \mathrm{TpG}$ and $\mathrm{CpG} \rightarrow \mathrm{CpA}$ mutations, with a higher penalty for insertions and deletions. We rooted the tree by selecting the oldest subfamily, that is, the subfamily with highest average divergence from its consensus sequence, as the root.

An implementation of our algorithm is available online at http://www.cs.ucsd.edu/ aprice/alu.html.

\section{References}

Arndt, P.F., Petrov, D.A., and Hwa, T. 2003. Distinct changes of genomic biases in nucleotide substitution at the time of mammalian radiation. Mol. Biol. Evol. 20: $1887-1896$.

Bailey, J.A., Liu, G., and Eichler, E.E. 2003. An Alu transposition model for the origin and expansion of human segmental duplications. Am. J. Hum. Genet. 73: 823-834.

Bao, Z. and Eddy, S.R. 2002. Automated de novo identification of repeat sequence families in sequenced genomes. Genome Res. 8: 1269-1276.
Batzer, M.A. and Deininger, P.L. 1991. A human-specific subfamily of Alu sequences. Genomics 9: 481-487.

2002. Alu repeats and human genomic diversity. Nat. Rev. Genet. 3: $370-379$.

Batzer, M.A., Deininger, P.L., Hellmann-Blumberg, U., Jurka, J., Labuda, D., Rubin, C.M., Schmid, C.W., Zietkiewicz, E., and Zuckerkandl, E. 1996. Standardized nomenclature for Alu repeats. J. Mol. Evol. 42: $3-6$.

Bishop, C. 1996. Neural networks for pattern recognition, pp. 189-190. Oxford University Press, Oxford, UK.

Britten, R.J., Baron, W.F., Stout, D.B., and Davidson, E.H. 1988. Sources and evolution of human Alu repeated sequences. Proc. Natl. Acad. Sci. 85: 4770-4774.

Deininger, P.L. and Batzer, M.A. 1995. SINE master genes and population biology. In The impact of short interspersed elements (SINEs) on the host genome (ed. R.J. Maraia), pp. 43-60. RG Landes, Georgetown, TX.

. 1999. Alu repeats and human disease. Mol. Genet. Metab. 67: 183-193.

Deininger, P.L. and Slagel, V.K. 1988. Recently amplified Alu family members share a common parental Alu sequence. Mol. Cell Biol. 8: $4566-4569$.

Deininger, P.L., Batzer, M.A., Hutchison, C.A., and Edgell, M.H. 1992. Master genes in mammalian repetitive DNA amplification. Trends Genet. 8: 307-311.

Dempster, A.P., Laird, N., and Rubin D.B. 1977. Maximum likelihood from incomplete data via the EM algorithm (with discussion). J. Roy. Stat. Soc., B 39: 1-38.

Dewannieux, M., Esnault, C. and Heidmann, T. 2003. LINE-mediated retrotransposition of marked Alu sequences. Nat. Genet. 35: 41-48.

International Human Genome Consortium. 2001. Initial sequencing and analysis of the human genome. Nature 409: 860-921.

Jurka, J. 1998. Repeats in genomic DNA: Mining and meaning. Curr. Opin. Struct. Biol. 8: 333-337.

. 2000. Repbase Update: A database and an electronic journal of repetitive elements. Trends Genet. 9: 418-420.

Jurka, J. and Milosavljevic, A. 1991. Reconstruction and analysis of human Alu genes. J. Mol. Evol. 32: 105-121.

Jurka, J. and Smith, T. 1988. A fundamental division in the Alu family of repeated sequences. Proc. Natl. Acad. Sci. 85: 4775-4778.

Keich, U. and Pevzner, P.A. 2002. Finding motifs in the twilight zone. Bioinformatics 18: 1374-1381.

Kruskal, J.B. 1956. On the shortest spanning tree of a graph and the traveling salesman problem. Proc. Am. Math. Soc. 7: 48-50.

Kurtz, S., Ohlebusch, E., Schleiermacher, C., Stoye, J., and Giegerich, R. 2000. Computation and visualization of degenerate repeats in complete genomes. In Proceedings of the Eighth International Conference on Intelligent Systems for Molecular Biology (ISMB-00), pp. 269-278. AAAI Press, San Diego, CA.

Labuda, D. and Striker, G. 1989. Sequence conservation in $A l u$ evolution. Nucleic Acids Res. 17: 2477-2491.

Leeflang, E.P., Liu, W.M., Hashimoto, C., Choudary, P.V. and Schmid, C.W. 1992. Phylogenetic evidence for multiple Alu source genes. J. Mol. Evol. 35: 7-16.

Leeflang, E.P., Liu, W.M., Chesnokov, I.N. and Schmid, C.W. 1993. Phylogenetic isolation of a human Alu founder gene: drift to new subfamily identity. J. Mol. Evol. 37: 559-565.

Lloyd, S.P. 1982. Least squares quantization in PCM. IEEE Trans. Inform. Theory 28: $129-137$

Matera, A.G., Hellmann, U., and Schmid, C.W. 1990a. A transpositionally and transcriptionally competent Alu subfamily. Mol. Cell Biol. 10: 5424-5432.

Matera, A.G., Hellmann, U., Hintz, M.F., and Schmid, C.W. 1990b. Recently transposed $\mathrm{Alu}$ repeats result from multiple source genes. Nucleic Acids Res. 18: 6019-6023.

Mathias, S.L., Scott, A.F., Kazazian Jr., H.H., Boeke, J.D., and Gabriel, A. 1991. Reverse transcriptase encoded by a human transposable element. Science 254: 1808-1810.

Milosavljevic, A., Haussler, D. and Jurka, J. 1989. Informed parsimonious inference of prototypical genetic sequences. In Proceedings of the Second Annual Workshop on Computational Learning Theory (eds. R. Rivest et al.), pp. 102-117. Morgan Kaufman, San Mateo, CA.

Nikaido, M., Rooney, A., and Okada, N. 1999. Phylogenetic relationships among cetartiodactyls based on insertions of short and long interspersed elements: Hippopotamuses are the closest extant relatives of whales. Proc. Natl. Acad. Sci. 96: 10261-10266.

Pevzner, P.A., Tang, H., and Tesler G. 2004. De novo repeat classification and fragment assembly. In Proceedings of the Eighth Annual International Conference on Computational Molecular Biology (RECOMB-04). ACM Press, San Diego, CA. 


\section{Price et al.}

Quentin, Y. 1988. The Alu family developed through successive waves of fixation closely connected with primate lineage history. J. Mol. Evol. 27: 194-202.

Rogers, J. 1983. Retroposons defined. Nature 301: 460

Salem, A.H., Ray, D.A., Xing, J., Callinan, P.A., Myers, J.S., Hedges, D.J., Garber, R.K., Witherspoon, D.J., Jorde, L.B., and Batzer, M.A. 2003. Alu elements and hominid phylogenetics. Proc. Natl. Acad. Sci. 100: $12787-12791$.

Schmid, C.W. 2003. Alu: a parasite's parasite? Nat. Genet. 35: 15-16.

Shen, M.R., Batzer, M.A., and Deininger, P.L. 1991. Evolution of the master Alu gene(s). J. Mol. Evol. 33: 311-320.

Takahashi, K., Nishida, M., Yuma, M., and Okada, N. 2001. Retroposition of the AFC family of SINEs before and during the adaptive radiation of cichlid fishes in Lake Malawi and related inferences about phylogeny. J. Mol. Evol. 53: 496-507.

Tatusova, T. and Madden, T. 1999. Blast 2 sequences-A new tool for comparing protein and nucleotide sequences. FEMS Microbiol. Lett. 174: $247-250$.

Ullu, E. and Weiner, A.M. 1985. Upstream sequences modulate the internal promoter of the human 7SL RNA gene. Nature

318: $371-374$
Volfovsky, N., Haas, B.J., and Salzberg, S.L. 2001. A clustering method for repeat analysis in DNA sequences. Genome Biol. 2: RESEARCH0027.

Watkins, W.S., Rogers, A.R., Ostler, C.T., Wooding, S., Bamshad, M.J., Brassington, A.M., Carroll, M.L., Nguyen, S.V., Walker, J.A., Prasad, B.V., et al. 2003. Genetic variation among world populations: Inferences from $100 \mathrm{Alu}$ insertion polymorphisms. Genome Res. 13: $1607-1618$.

Willard, C., Nguyen, H.T., and Schmid, C.W. 1987. Existence of at least three distinct Alu subfamilies. J. Mol. Biol. 26: 180-186.

\section{Web site references}

http://repeatmasker.org; RepeatMasker.

http://www.cs.ucsd.edu/ aprice/alu.html; implementation of our algorithm.

Received April 18, 2004; accepted in revised form August 14, 2004. 


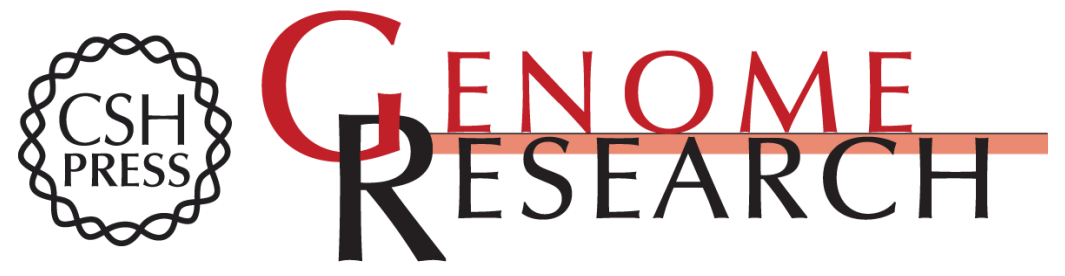

\section{Whole-genome analysis of Alu repeat elements reveals complex evolutionary history}

Alkes L. Price, Eleazar Eskin and Pavel A. Pevzner

Genome Res. 2004 14: 2245-2252

Access the most recent version at doi:10.1101/gr.2693004

Supplemental Material

References

License

Email Alerting Service
http://genome.cshlp.org/content/suppl/2004/10/06/14.11.2245.DC1

This article cites 35 articles, 8 of which can be accessed free at: http://genome.cshlp.org/content/14/11/2245.full.html\#ref-list-1

Receive free email alerts when new articles cite this article - sign up in the box at the top right corner of the article or click here.

\section{Affordable, Accurate Sequencing.}

